J Electrocardiol. 2014 ; 47(3): 356-361. doi:10.1016/j.jelectrocard.2014.03.002.

\title{
Short-term repeatability of electrocardiographic Tpeak-Tend and QT intervals
}

\author{
Kapuaola S Gellert, MPH${ }^{\star}$, Pentti Rautaharju, MD, PhD ${ }^{\dagger}$, Michelle L Snyder, PhD, MPH${ }^{\star}$, Eric \\ A Whitsel, MD, MPH ${ }^{\star}, \#$, Kunihiro Matsushita, MD, $\mathrm{PhD}^{\ddagger}$, Gerardo Heiss, MD, PhD ${ }^{\star}$, and \\ Elsayed Z Soliman, MD ${ }^{\dagger}, \S$ \\ *Department of Epidemiology, Gillings School of Global Public Health, University of North \\ Carolina at Chapel Hill, Chapel Hill, NC \\ †Department of Public Health Sciences, Epidemiological Cardiology Research Center \\ (EPICARE), Wake Forest University School of Medicine, Winston Salem, NC \\ \#Department of Medicine, School of Medicine, University of North Carolina at Chapel Hill, Chapel \\ Hill, NC \\ ‡Department of Epidemiology, Johns Hopkins Bloomberg School of Public Health, Baltimore, MD \\ §Department of Medicine, Section on Cardiology, Wake Forest School of Medicine, Winston \\ Salem, NC
}

\begin{abstract}
Background-The electrocardiographic (ECG) Tpeak-Tend interval (TpTe) is associated with arrhythmias and sudden cardiac death. TpTe offers a supplementary measure for the QT interval (QT), yet its repeatability has not been established.

Purpose-Evaluate short-term repeatability of TpTe and QT.

Methods-Four ECGs were obtained on sixty participants. The sources of variation, intra-class correlation coefficient (ICC) - an index of reproducibility, and minimal detectable change (MDC) were estimated for TpTe and QT. The impact of repeated measurements on repeatability was estimated for a hypothetical clinical trial designed to detect drug-induced prolongation of $\mathrm{TpTe}$ and QT.
\end{abstract}

Results-We used heart rate-adjusted QT [(QT)a] but TpTe in the study group was rateinvariant. The ICC [95\% confidence interval $(\mathrm{CI})]$ was $0.77(0.69,0.85)$ for TpTe, $0.75(0.65$, $0.85)$ for QT and $0.68(0.57,0.79)$ for $(\mathrm{QT}) \mathrm{a}$. The MDC (ms) was 21,32 and 26 for TpTe, QT and (QT)a respectively.

(c) 2014 Elsevier Inc. All rights reserved.

Corresponding Author: Kapuaola S. Gellert, 137 E. Franklin St, Suite 306, Chapel Hill, NC, 27514, Phone: (919) 966-2393, Fax: (919) 966-9800, kapuaola@email.unc.edu.

Publisher's Disclaimer: This is a PDF file of an unedited manuscript that has been accepted for publication. As a service to our customers we are providing this early version of the manuscript. The manuscript will undergo copyediting typesetting, and review of the resulting proof before it is published in its final citable form. Please note that during the production process errors may be discovered which could affect the content and all legal disclaimers that apply to the journal pertain. 
Conclusion-TpTe has excellent repeatability supporting its use as a supplement to QT in observational and clinical studies.

\section{Keywords}

Electrocardiogram; QT; TpTe interval; Repeat variation T

\section{Introduction}

The Tpeak-Tend interval (TpTe) from the resting standard 12-lead electrocardiogram (ECG) is a novel measure of myocardial repolarization, reported to be associated with incident ventricular arrhythmia, heart failure, and sudden cardiac death (SCD) (1-4). Prolongation of TpTe represents a period of potential vulnerability to reentrant arrhythmias offering a supplement to the traditional QT interval (QT) (5) in the setting of the established associations between QT prolongation with total, cardiovascular and sudden cardiac death (1). Although widely used to quantify ventricular repolarization abnormalities, QT has limitations and varying recommendations have been issued for an appropriate heart rate correction (6-8). An increased interest in other measures of myocardial repolarization has thus emerged, including TpTe (5). The peak of the $\mathrm{T}$ wave occurs when the largest number of left ventricular lateral wall myocytes is at phase 3 of their repolarization and the uncanceled repolarization wave front is largest according to the infinite-medium model of ventricular repolarization $(8,9)$. This assertion is supported by electrophysiological evidence and electrocardiographic potential theory applied to the generation of the T wave (10-12). Opthof et al. (13) have pointed out that the interval from $\mathrm{T}$ wave onset to Te reflects total dispersion of repolarization, therefore providing more comprehensive clinical information of arrhythmic risk.

The association of TpTe with ventricular arrhythmia and SCD and its consideration as a supplement for QT makes it pertinent to evaluate the repeatability of TpTe, which to our knowledge has not been addressed. The aim of our study was to characterize the repeatability and main sources of variability of $\mathrm{TpTe}$, and to contrast these properties with those of QT.

\section{Methods}

\section{Study participants and protocol}

Study participants included sixty male and female volunteers free of cardiovascular symptoms recruited in Chapel Hill, NC. Details of study participant recruitment were described elsewhere (15). Briefly, efforts were made to match the age, gender and racial characteristics of the volunteer group to that of the Atherosclerosis Risk in Communities (ARIC) cohort at baseline and included participants between 45-64 years of age, free of diabetes, hypertension, emphysema, chronic obstructive pulmonary disease, congestive heart failure, renal disease, a pacemaker, and were not taking class I or III antiarrhythmics. Informed consent was obtained from all participants. This study was approved by the University of North Carolina (UNC) School of Medicine institutional review board. All examinations were conducted at the General Clinical Research Center of the UNC Hospitals. 


\section{Electrocardiographic methodology}

The protocol used by the ARIC study was followed for electrode positioning, room condition, data collection and transfer (15). Following identical protocol at both study visits, two sets of ECGs were obtained on the same study participant at each visit. Four digital 12lead ECGs (ECG1, ECG2, ECG3, and ECG4) were obtained by one of four trained and certified technicians at two different examination visits [two ECGs per visit, ten days apart on average, standard deviation (SD)=four days] both of which were conducted between 7:30 and 11:30 am, following a 12-hour period during which participants were asked not to consume anything other than water and to refrain from smoking and intense physical activity. The two ECG recordings at each study examination were separated by one to two minutes and were performed without removal of the electrodes. The trained and certified technicians positioned Kendall Q-Trace $5400 \mathrm{Ag} / \mathrm{AgCl}$ electrodes (Ludlow Co, Chicopee, MA) using the E-V6 half-point method and a standardized protocol for placing electrodes on women after a 15-minute supine rest (16). ECGs were recorded using a MAC Personal Cardiographer (Marquette Electronics, Inc., Jupiter, FL). ECGs were digitized at 250 samples per second per lead and sent via modem after each recording session to the Epidemiology Cardiology Research (EPICARE) Center at the Wake Forest University School of Medicine in Winston-Salem, NC. The EPICARE reading center, masked to participant identity and previous ECG readings, processed the ECGs with the Marquette program GE 12SL software 2001 version (GE, Milwaukee, WI). All ECGs were visually reviewed, and all measurements were validated using interactive software. Simultaneous ECGs were displayed to the reviewers expanded on the graphics screen with computerplaced global time lines at QRS onset, QRS end and Tend. The reviewers had the option either to accept computer time lines or to move them to a correct location if in error. The algorithms for detection of Tend in the GE 12SL program are based on combined slope and amplitude thresholds (17). Simultaneous TpTe was measured as the interval (ms) from the peak to the end of the global $\mathrm{T}$ wave (the $\mathrm{T}$ wave vector magnitude curve).

\section{Statistical analysis}

Our analysis excluded three participants from the original sample size of sixty three; two with extreme outliers ( $\mathrm{TpTe}<50 \mathrm{~ms}$ and QT $>480 \mathrm{~ms}$ ) between 2.5 and $4 \mathrm{SD}$ away from the mean, and one for evidence of an arrhythmia indicated by frequent ventricular premature beats. We calculated the frequency and percent of selected categorical demographic variables and the mean and standard deviation of interval-scale measures. Linear formulas were used to calculate heart rate-adjusted QT as $(\mathrm{QT}) \mathrm{a}=\mathrm{QTe}+127 *(1-\mathrm{RR})$ for men and $(\mathrm{QT}) \mathrm{a}=\mathrm{QTe}+136 *(1-\mathrm{RR}$ for women). Bazett rate-adjusted QT $(\mathrm{QT}) \mathrm{bz}$ was calculated as $\left[\mathrm{QTe} /(\mathrm{RR})^{1 / 2}\right]$ (18). Mean values and SD for TpTe, (QT)a and (QT)bz at each study visit were calculated. We used random-effects models: $Y_{i j k}=\mu+T p T e+V_{j(i)}=e_{k(i j)}$ where $\mu$ is the intercept, $i=1,2,3$ to the $60^{\text {th }}$ participant, $\mathrm{j}$ is either visit 1 of visit 2 and $\mathrm{k}$ is the first or second ECG recording to estimate sources of variation in TpTe and QT [between-participant $\left(\sigma_{\mathrm{p}}^{2}\right)$, between-visit $\left(\sigma_{b v}^{2}\right)$, and within-visit $\left.\left(\sigma_{w v}^{2}\right)\right]$. Two assumptions were that within-visit variation is represented by the residual variation in the model, between-participant variation was the same for all participants, and within-visit variation was the same for all visits and all participants, as described previously (6). 


\section{Intraclass correlation coefficient and standard error of measurement}

The intraclass correlation coefficient (ICC) was defined as the ratio of between-participant variance to total variance observed in a repeated measurements study.

$I C C=\left(\frac{\sigma_{\mathrm{p}}^{2}}{\sigma_{\text {Total }}^{2}}=\frac{\sigma_{\mathrm{p}}^{2}}{\sigma_{\mathrm{p}}^{2}+\sigma_{b v}^{2}+\sigma_{w v}^{2}}\right)$ where $\sigma_{\mathrm{p}}^{2}$ is the variance between participants, $\sigma_{b v}^{2}$ is the variance between visits, and $\sigma_{w v}^{2}$ is the variance within visit. Thus high ICC indicates that the variation of the parameter of interest is mainly due to variation between participants suggesting high repeatability within participants. Variance between technicians was

negligible. We also calculated the standard error of measurement $(S E M)=\sqrt{\sigma_{b v}^{2}+\sigma_{w v}^{2}}$. The minimal detectable change $(\mathrm{MDC}=\mathrm{SEM} * \sqrt{2} * 1.96)$ was calculated with $95 \%$ confidence intervals (CI).

\section{$95 \%$ Confidence intervals}

We also estimated the impact of repeated measurements of TpTe and QT on the precision of the TpTe and QT measurements for a hypothetical clinical trial as a function of the number of measurements, $\mathrm{n}$, and the sample size of such a trial, N. Since no previous requirement was issued for designing clinical trials powered to detect TpTe prolongation that would identify possible side effects of a drug, we used the requirement of 5-ms that was established for QT (6). This aspect of the repeatability analysis allows us to examine the ability to detect side effects associated with prolongation of TpTe and QT that may be encountered during a clinical trial especially those side effects resulting from drugs. The 95\% CIs were calculated for the "true" change in the mean TpTe and QT intervals in a hypothetical clinical trial averaged over replicate measures and the SD (estimate of change $\pm 1.96 \mathrm{SD}$ ).

The SD for this estimate of change was calculated the same way for QT and TpTe assuming a clinical trial with 2 arms where X' was the mean postdrug-predrug change in TpTe was the mean postdrug-predrug change in $\mathrm{TpTe}$ in the treatment group, and $\mathrm{X}^{\prime} \mathrm{T}=0$ was the mean postdrug-predrug change in TpTe in the control group. The variance (Var) of the difference in TpTe change between the treatment and control group was: $\operatorname{Var}\left(X^{\prime}{ }_{T=1}-X^{\prime} T=0\right)=\operatorname{Var}$ $\left(X^{\prime} T=1\right)+\operatorname{Var}\left(X_{T=0}^{\prime}\right)$. Based on the assumption that the variances in the treatment and control group are identical, the variance of the difference in TpTe change between the treatment and control group was calculated as the following: $4 *$ within-participant variance/ $(n * N)$ where $n$ is the number of replicate measures and $N$ is the sample size.

Similar to the calculations above that were for group level changes, the $95 \% \mathrm{CI}$ for individual changes were calculated as the estimate of change $\pm 1.96 * \sqrt{ } 2 *$ intra-individual SD/ $\mathrm{V}$, where $\mathrm{n}$ is the number of repeated measurements. All data were analyzed using SAS version 9.3 (SAS Institute, Inc., Cary, NC).

\section{Results}

\section{Characteristics of study participants}

The demographic and clinical characteristics of study participants are shown in Table 1. Participants included equal numbers of males and females who mostly self-identified as 
white (68\%), with a mean age of $52 \pm 5$ years, and an average body mass index (BMI) of $27 \pm 5$. Medication use was low; anticholinergic (8\%), beta-blocker (2\%), diuretic (6\%), selective serotonin reuptake inhibitor $(17 \%)$, sympathomimetic (7\%), and thyroid medication (5\%). A first degree atrioventricular block was observed in $15 \%$ of the study participants. Non-fasting/smoking twelve-hour pre-examination was rare at either visit, $7 \%$.

We reported (QT)a but TpTe in the study group was rate-invariant. The mean values and the standard deviations of TpTe were similar across all four measurements at the two visits. The mean values and the standard deviations of QT and (QT)a were also similar across all four measurements at the two visits with smaller values overall for (QT)a compared to QT (Table $2)$. The absolute between-visit difference was $(7 \pm 5 \mathrm{~ms})$ for TpTe and was similar to the within-visit difference ( $8 \pm 6 \mathrm{~ms}$ ). The absolute value of the between-visit difference ( $7 \pm 6$ $\mathrm{ms})$ for QT was also similar to the within-visit difference $(6 \pm 6 \mathrm{~ms})$. The absolute value of the between-visit difference and the within-visit difference for $(\mathrm{QT})$ a respectively were $(9 \pm$ $8 \mathrm{~ms})$ and $(7 \pm 5 \mathrm{~ms})$ (Table 2$)$.

Components of measurement error are listed in Table 3. Between-participant variation accounted for $77 \%$ of the total variation in TpTe, $75 \%$ of QT, $60 \%$ of (QT)a, and $69 \%$ of (QT)bz. Within-visit variation for TpTe accounted for a large proportion of the total variation than the between-visit variation (20\% vs. $3 \%)$ in the random effects model. The within-visit variation for (QT)a. also accounted for more (27\%) than the between-visit $(13 \%)$ variation.

TpTe had an ICC of 0.77 and the MDC between two measures was $21 \mathrm{~ms}$. The ICC for (QT)a and (QT)bz were 0.60 and 0.68 respectively. The MDC was $32 \mathrm{~ms}$ for (QT)a and 29 ms for QTbz (Table 3).

Table 4 shows the impact of repeated measurements of TpTe and QT on precision. The $95 \%$ CIs for a mean $5 \mathrm{~ms}$ effect on TpTe and QT was calculated as a function of the number of measurements $(\mathrm{n})$ and the total sample size $(\mathrm{N})$. The more precise CIs were seen when the number of measurements and number of participants was larger. For example, when two ECGs are available from baseline and there are 60 enrolled participants in a clinical trial, if the placebo-corrected increase was $5 \mathrm{~ms}$, the $95 \% \mathrm{CI}$ around this estimate for TpTe would be between 2 and $8 \mathrm{~ms}$ and between 1 and $9 \mathrm{~ms}$ for (QT)a. Calculations of these estimations at the individual level show much larger imprecision (Table 5). For example, when two ECGs are available from baseline for a participant, this estimate ranges between 5 and $35 \mathrm{~ms}$ for TpTe and between 2 and $38 \mathrm{~ms}$ for (QT)a (Table 5).

\section{Discussion}

The TpTe and QT intervals from the 12-lead ECG have excellent short-term repeatability as the between-participant variability accounts for most of the total variability. Our mean values for TpTe were similar to those reported in a study of a younger population $(92 \pm 11$ $\mathrm{ms}$ for women and $94 \pm 10 \mathrm{~ms}$ for men) by Haarmark et al (19). The results of our repeatability analysis were similar for $\mathrm{TpTe}$ and (TpTe)a (not shown). This similarity between $\mathrm{TpTe}$ and (TpTe)a was reported in a study of the electrocardiographic predictors of 
coronary heart disease and SCD in ARIC participants where investigators reported that $\mathrm{TpTe}$ in CVD-free participants was practically independent from heart rate (8).

Short-term variability in TpTe and QT like that of other ECG markers used to characterize ventricular repolarization is mostly explained by two main factors: biological variability and measurement error (6). Between-participant variability was the largest source of variability for TpTe and (QT)a. Within-visit variability accounted for slightly more variation than the between-visit for TpTe and (QT)a, which is likely related to the model assumption that the residual variation is due to within-visit variation and to the difficulty of partitioning withinvisit from between-visit variation. This suggests that little variation was added when relating two ECGs from different visits compared to relating two ECGs from the same visit. The process-related variability was minimized by the standard ARIC protocol that was followed for electrode positioning, room condition, data collection and transfer (15).

The traditional measure of myocardial repolarization is the QT interval because its prognostic value is associated with arrhythmic risk, specifically torsades de pointes (TdP), a polymorphic ventricular tachycardia (20). TdP is the most serious adverse effect of drug therapy (21) and it is associated with rates of mortality between 10-17\% (22). Because of these adverse associations, presence of QT prolongation has led to termination of development and withdraw from the market of many drugs, rejection of new drugs, and restrictions on prescribing new and old drugs $(22,23)$. Although QT prolongation has been widely used as a predictor of arrhythmic risk, it has more recently been described as being an imperfect measure (22). TpTe has been shown to be associated with SCD as the odds of prolonged $\mathrm{TpTe}$ were reported to be greater prior to and unrelated to SCD events, compared to controls from the same geographic area who had coronary artery disease (3). The limitations of QT prolongation as a surrogate of TdP and other VT and VF and as a measure to predict arrhythmic risk (22) are related to the interest in new measures of repolarization. TpTe has emerged as a novel measure of myocardial repolarization and a supplement to QT conveying information on arrhythmic risk (24).

To our knowledge, this is the first study to evaluate the short-term repeatability of $\mathrm{TpTe}$ from the standard 12-lead ECG. Another study using the same study population as ours, but without excluding participants with extreme outliers for TpTe and QT or evidence of an arrhythmia $(\mathrm{n}=63)$, reported that QT is a repeatable measure of ventricular repolarization given that between-participant variation $(86 \%)$ was the main source of the variation between repeated measurements (5). The ICC for TpTe and QT were all close to unity and therefore more likely that the participants in our study and those in the study of QT repeatability displayed consistent measurements observed across visits compared to differences in measurements observed across participants.

The MDC estimate for TpTe was comparable to its SDs. Similarly, the MDC for (QT)a was comparable to its SD. Examination of the impact of repeated measurements on precision showed consistent results for TpTe and (QT)a, where gains in precision were seen with increasing numbers of enrolled participants. 
The relatively small sample size is a limitation of our study. Another limitation was that our study design did not allow for an estimation of technician variation, however the variance due to technician is likely negligible as reported by Schroeder and Vaidean $(6,25)$. The strengths of our study include the standardized ECG measurement protocol conducted at two time points and the central training of the technicians.

The high repeatability and low MDC between measurements support the use of TpTe as a supplement to QT in observational and clinical studies to evaluate myocardial repolarization characteristics related to ventricular arrhythmia, heart failure and sudden cardiac death.

\section{Acknowledgments}

The Atherosclerosis Risk in Communities Study is carried out as a collaborative study supported by National Heart Lung, and Blood Institute contracts (HHSN268201100005C, HHSN268201100006C, HHSN268201100007C, HHSN268201100008C, HHSN268201100009C, HHSN268201100010C, HHSN268201100011C, and HHSN268201100012C). The authors thank the study participants for their important contribution. K.S.G. and M.L.S were supported by the National Heart Lung, and Blood Institute T32 training grant HL-007055.

\section{References}

1. Zhang Y, Post WS, Blasco-Colmenares E, et al. Electrocardiographic QT interval and mortality: a meta-analysis. Epidemiology. 2011; 22(5):660-70.10.1097/EDE.0b013e318225768b [PubMed: 21709561]

2. Kanters JK, Haarmark C, Vedel-Larsen E, et al. T(peak)T(end) interval in long QT syndrome. J Electrocardiol. 2008; 41(6):603-8.10.1016/j.jelectrocard.2008.07.024 [PubMed: 18822425]

3. Panikkath R, Reinier K, Uy-Evanado A, et al. Prolonged Tpeak-to-tend interval on the resting ECG is associated with increased risk of sudden cardiac death. Circ Arrhythm Electrophysiol. 2011; 4(4): 441-7.10.1161/CIRCEP.110.960658 [PubMed: 21593198]

4. Castro Hevia J, Antzelevitch C, Tornes Barzaga F, et al. Tpeak-Tend and Tpeak-Tend dispersion as risk factors for ventricular tachycardia/ventricular fibrillation in patients with the Brugada syndrome. J Am Coll Cardiol. 2006; 47(9):1828-34.10.1016/j.jacc.2005.12.049 [PubMed: 16682308]

5. Antzelevitch $\mathrm{C}$. T peak-Tend interval as an index of transmural dispersion of repolarization. Eur $\mathbf{J}$ Clin Invest. 2001; 31(7):555-7. [PubMed: 11454006]

6. Vaidean GD, Schroeder EB, Whitsel EA, et al. Short-term repeatability of electrocardiographic spatial T-wave axis and QT interval. J Electrocardiol. 2005; 38(2):139-47.10.1016/j.jelectrocard. 2004.09.020 [PubMed: 15892024]

7. Roden DM. Drug-induced prolongation of the QT interval. N Engl J Med. 2004; 350(10):101322.10.1056/NEJMra032426 [PubMed: 14999113]

8. Rautaharju PM, Zhang ZM, Warren J, et al. Electrocardiographic predictors of coronary heart disease and sudden cardiac deaths in men and women free from cardiovascular disease in the atherosclerosis risk in communities study. J Am Heart Assoc. 2013; 2(3):e000061.10.1161/JAHA. 113.000061 [PubMed: 23723252]

9. Rautaharju PM, Zhang ZM, Haisty WK Jr, et al. Electrocardiographic Predictors of Incident Heart Failure in Men and Women Free From Manifest Cardiovascular Disease (from the Atherosclerosis Risk in Communities [ARIC] Study). Am J Cardiol. 201310.1016/j.amjcard.2013.05.011

10. Yan GX, Antzelevitch C. Cellular basis for the normal T wave and the electrocardiographic manifestations of the long-QT syndrome. Circulation. 1998; 98(18):1928-36. [PubMed: 9799215]

11. Xia Y, Liang Y, Kongstad O, et al. In vivo validation of the coincidence of the peak and end of the $\mathrm{T}$ wave with full repolarization of the epicardium and endocardium in swine. Heart Rhythm. 2005; 2(2):162-9.10.1016/j.hrthm.2004.11.011 [PubMed: 15851290] 
12. Zhu TG, Patel C, Martin S, et al. Ventricular transmural repolarization sequence: its relationship with ventricular relaxation and role in ventricular diastolic function. Eur Heart J. 2009; 30(3):37280.10.1093/eurheartj/ehn585 [PubMed: 19147608]

13. Opthof T, Coronel R, Wilms-Schopman FJ, et al. Dispersion of repolarization in canine ventricle and the electrocardiographic T wave: Tp-e interval does not reflect transmural dispersion. Heart Rhythm. 2007; 4(3):341-8.10.1016/j.hrthm.2006.11.022 [PubMed: 17341400]

14. Xia Y, Liang Y, Kongstad O, et al. Tpeak-Tend interval as an index of global dispersion of ventricular repolarization: evaluations using monophasic action potential mapping of the epi- and endocardium in swine. J Interv Card Electrophysiol. 2005; 14(2):79-87.10.1007/ s10840-005-4592-4 [PubMed: 16374554]

15. The Atherosclerosis Risk in Communities (ARIC) Study: design and objectives. The ARIC investigators. Am J Epidemiol. 1989; 129(4):687-702. [PubMed: 2646917]

16. Rautaharju PM, Wolf HK, Eifler WJ, et al. A simple procedure for positioning precordial ECG and VCG electrodes using an electrode locator. J Electrocardiol. 1976; 9(1):35-40. [PubMed: 1245809]

17. Hnatkova K, Gang Y, Batchvarov VN, et al. Precision of QT interval measurement by advanced electrocardiographic equipment. Pacing Clin Electrophysiol. 2006; 29(11):1277-84.10.1111/j. 1540-8159.2006.00532.x [PubMed: 17100684]

18. Bazett J. An analysis of time relations of electrocardiograms. Heart. 1920; 7:353-367. Heart. 1920;7:353-67.

19. Haarmark C, Graff C, Andersen MP, et al. Reference values of electrocardiogram repolarization variables in a healthy population. J Electrocardiol. 2010; 43(1):31-9. doi: http:// dx.doi.org.libproxy.lib.unc.edu/10.1016/j.jelectrocard.2009.08.001. [PubMed: 19740481]

20. Khan IA, Win MT, Bali AJ, et al. Torsades de pointes: a case with multiple variables. Am J Emerg Med. 1999; 17(1):80-5. [PubMed: 9928709]

21. Morganroth J. Relations of QTc prolongation on the electrocardiogram to torsades de pointes: definitions and mechanisms. Am J Cardiol. 1993; 72(6):10B-3B.

22. Shah RR, Hondeghem LM. Refining detection of drug-induced proarrhythmia: QT interval and TRIaD. Heart Rhythm. 2005; 2(7):758-72.10.1016/j.hrthm.2005.03.023 [PubMed: 15992736]

23. Food and Drug Administration, HHS. International Conference on Harmonisation; guidance on E14 Clinical Evaluation of QT/QTc Interval Prolongation and Proarrhythmic Potential for NonAntiarrhythmic Drugs; availability. Notice. Fed Regist. 2005; 70(202):61134-5. [PubMed: 16237860]

24. Huikuri HV, Castellanos A, Myerburg RJ. Sudden death due to cardiac arrhythmias. N Engl J Med. 2001; 345(20):1473-82.10.1056/NEJMra000650 [PubMed: 11794197]

25. Schroeder EB, Whitsel EA, Evans GW, et al. Repeatability of heart rate variability measures. J Electrocardiol. 2004; 37(3):163-72. [PubMed: 15286929] 


\section{Table 1}

Characteristics of the study participants $(n=60)$

\begin{tabular}{ll} 
Variable & Number $(\%)$ or Mean \pm standard deviation \\
\hline Female & $30(50)$ \\
Non-white & $19(32)$ \\
Age (years) & $52 \pm 5$ \\
Body mass index $\left(\mathrm{kg} / \mathrm{m}^{2}\right)$ & $*$ \\
Medication Use & $27 \pm 5$ \\
$\quad$ Anticholinergic & \\
$\quad$ Beta-Blocker & $5(8)$ \\
$\quad$ Diuretic & $1(2)$ \\
Selective serotonin reuptake inhibitor (SSRI) & $4(7)$ \\
$\quad$ Sympathomimetic & $10(17)$ \\
$\quad$ Thyroid medication & $4(7)$ \\
Atrioventricular block (per subject) & $3(5)$ \\
Non-fasting/smoking <12 hours before either visit & $4(7)$ \\
\hline * & $9(15)$ \\
$\mathrm{n}=59$ &
\end{tabular}




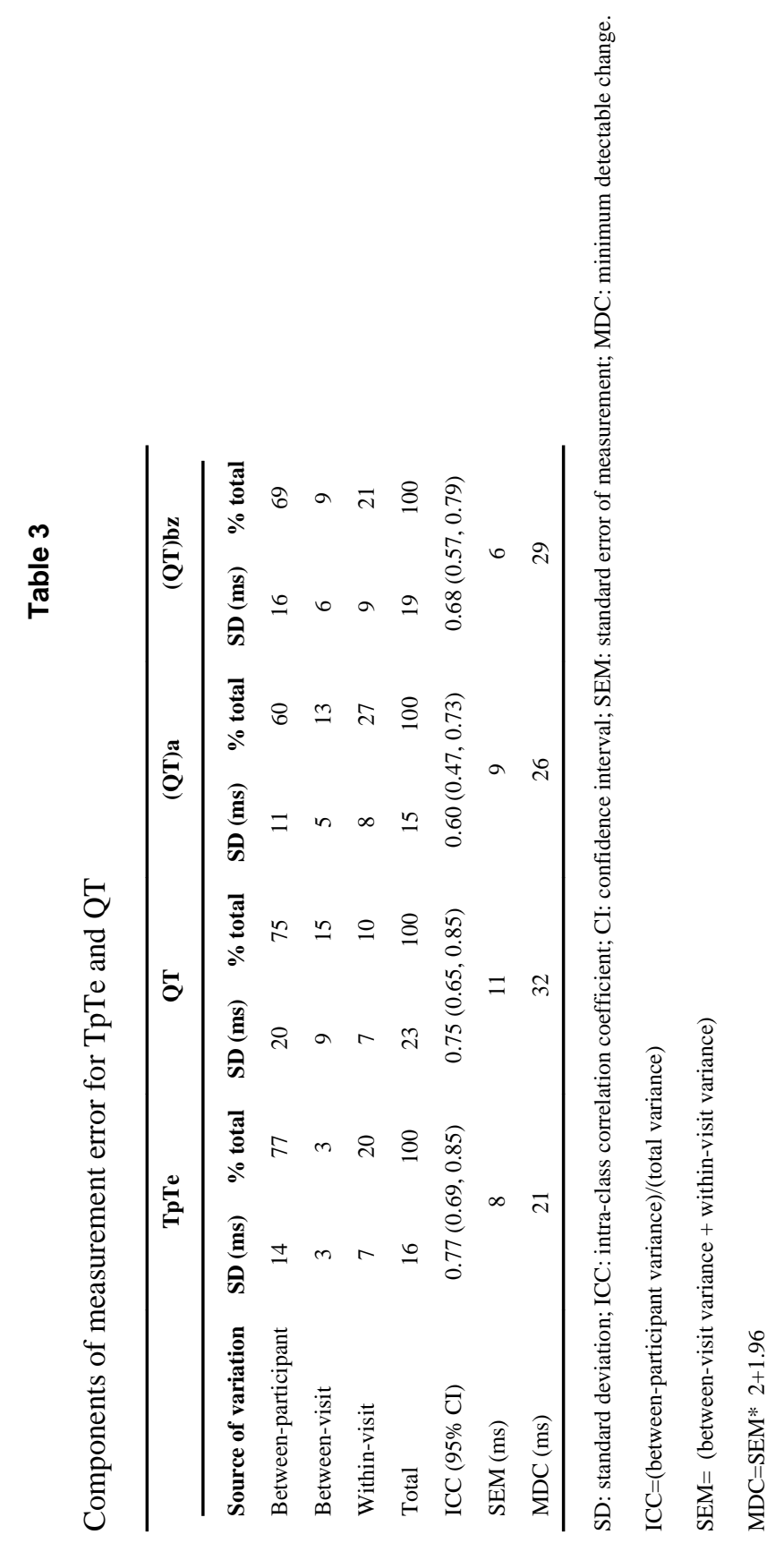

J Electrocardiol. Author manuscript; available in PMC 2015 May 01. 


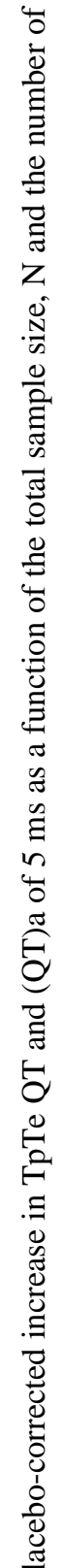

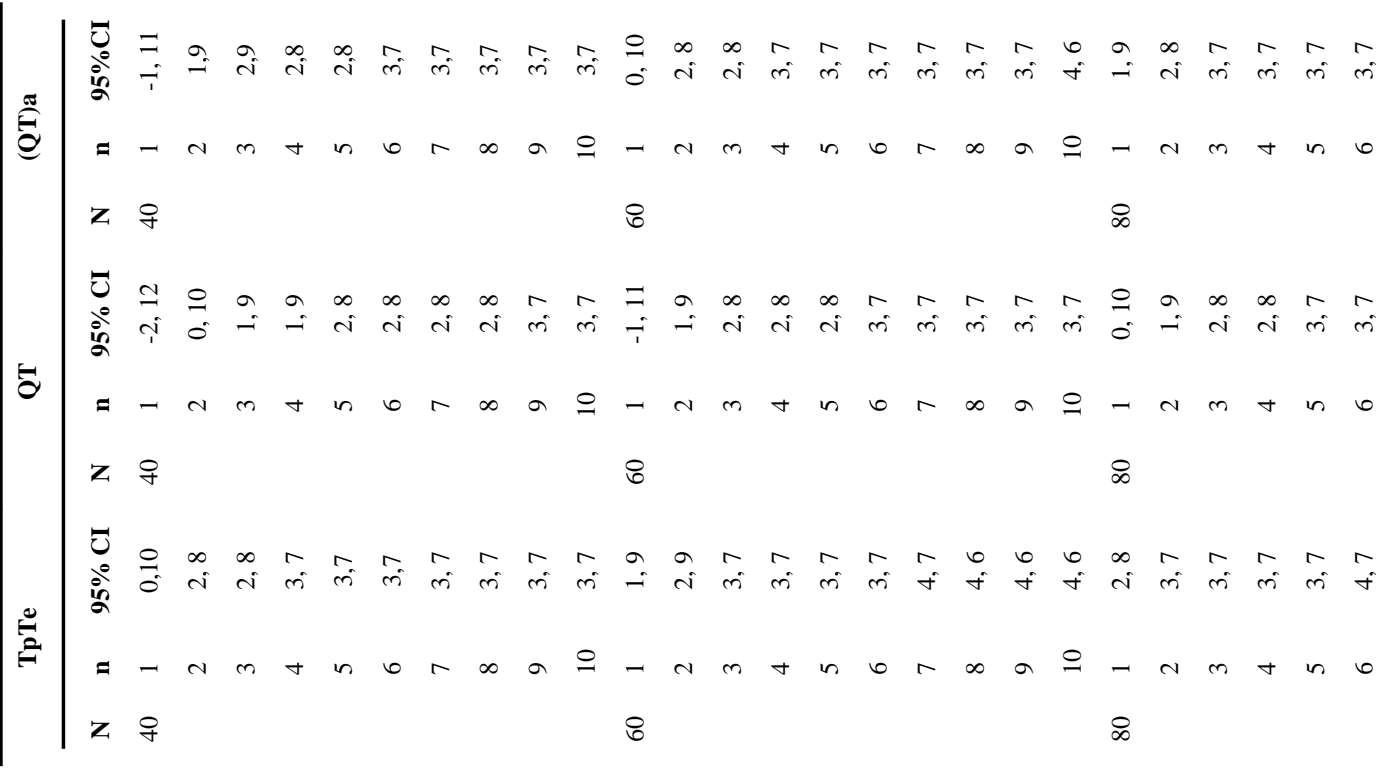
J Electrocardiol. Author manuscript; available in PMC 2015 May 01. 


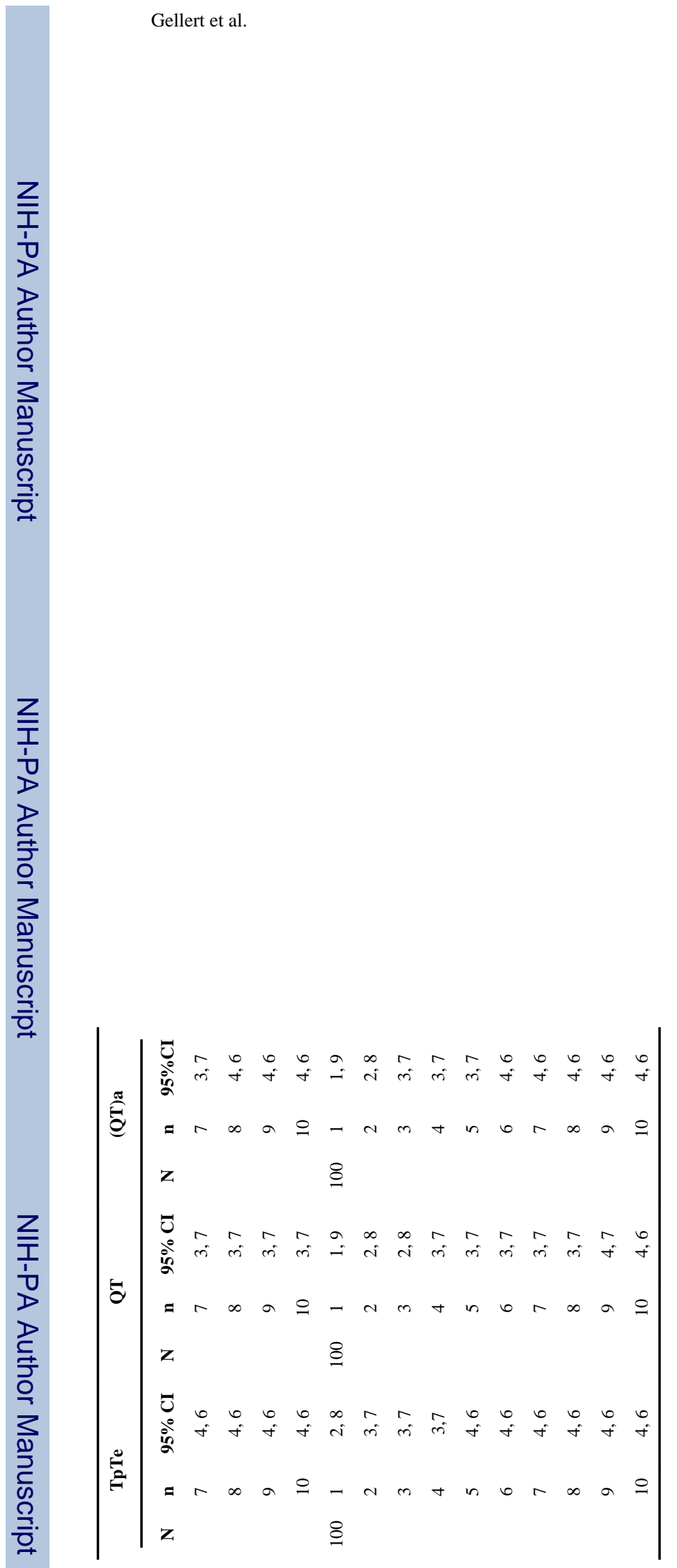

Page 13 


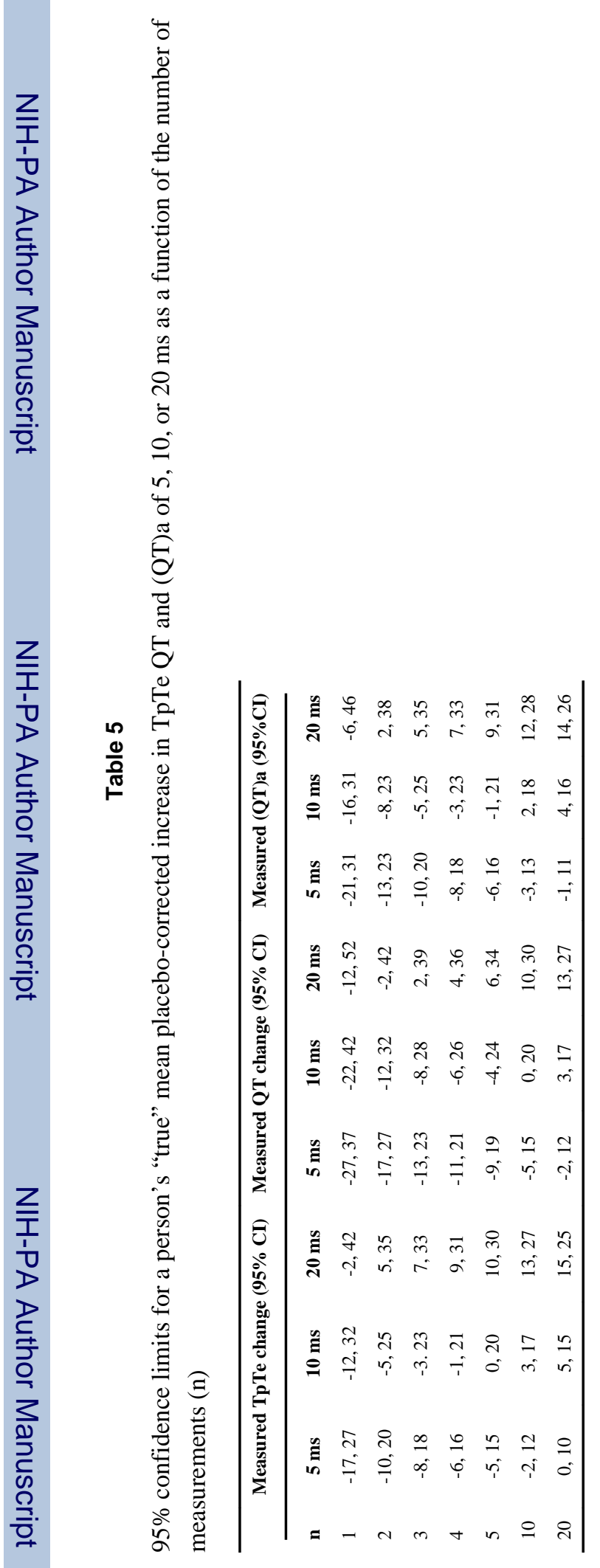

